\title{
On Fundamentality of Heat
}

\author{
Alireza Jamali* \\ Senior Researcher \\ Natural Philosophy Department, Hermite Foundation ${ }^{\dagger}$ \\ alireza.jamali.mp@gmail.com
}

September 18, 2021

\begin{abstract}
Motivated by the well-known contradiction of special relativity and the heat equation, a wave equation for temperature scalar field is presented that also resolves the old controversy of (Lorentz) transformation of temperature and entropy. After showing that the current dogma of temperature and entropy being emergent concepts is based on but a logical fallacy, it is proposed that single particles posses entropy. This principle of fundamentality of entropy is then shown to be compatible with the equipartition theorem by yielding corrections in the quantum gravity regime.
\end{abstract}

Keywords - heat equation, relativistic heat equation, emergence of entropy, equipartition theorem

\section{Contents}

1 Introduction

2 Wave Equation for Temperature Scalar Field 6

3 Covariant theory $\quad 7$

3.1 Onsager four-vector . . . . . . . . . . . . 7

3.2 Constructing Temperature four-vector . . . . . . . . 7

3.3 Onsager field tensor . . . . . . . . . . . . . . . . . . . . . . 8

3.4 Lagrangian density . . . . . . . . . . . . . . . 8

${ }^{*}$ Corresponding author

†3rd Floor - Block No. 6 - Akbari Alley - After Dardasht Intersection - Janbazané Sharghi - Tehran - Iran 
4 Implications $\quad \mathbf{8}$

4.1 Temperature and Entropy of a moving body . . . . . . . . 8

4.2 Entropy of single particles . . . . . . . . . . . 8

4.2.1 Induction on de Broglie's methodology . . . . . . . . 9

4.3 Principle of Maximum Temperature . . . . . . . . . . . . 9

4.4 Corrections to the equipartition theorem . . . . . . . . . . 11

5 Conclusion

\section{Introduction}

It has been 115 years since annus mirabilis when the last conceptual'philosophical'1 progress was made in foundations of thermodyanmics and still a coherent overarching theory of heat is missing. Countless attempts has been made in various directions focusing on separate problems of the theory without any attempt towards an all-encompassing theory which solves all the well-known problems at once. Perhaps now is the time to recall the Einsteinian lesson that resolution of a persistent problem is not more persistence but rebellion; a rebellion that questions the dogmas that are thought of as indubitable truths. There are two persistent fundamental problems in our current understanding of heat:

- Propagation of heat as governed by the heat equation is incompatible with special relativity[1].

- Lorentz transformation of temperature and entropy is not decisively known and is at best controversial[2].

The dogma that has shaped all the attempts directed at solving these problems is that temperature and entropy are emergent concepts and single particles do not posses entropy or temperature. As a result of believing in this dogma all such attempts has become messy and blind, involving all sorts of arbitrarinesses. It is exactly this dogma that I am going to rebel against in this paper. One of my contributions in this paper is to show that 'temperature and entropy are emergent' is indeed a dogma, hence addition of a third serious problem to the problems mentioned above. A dogma can be defined to be a statement -held by majority of a societythat is logically flawed. In order to see how 'temperature and entropy are emergent' is a dogma let us review the reason behind it: According to statistical mechanics, the temperature of a system in equilibrium is defined $[3,4,5]$ by

$$
\frac{1}{T}:=\frac{\partial S}{\partial E}
$$

where $S=k_{B} \log W(E)$. This established mathematical defintion results in other interpretations of said defintion, such as defining temperature by the average kinetic energy of particles

$$
\frac{3}{2} N k_{B} T=\frac{1}{2}\left\langle m v^{2}\right\rangle .
$$

\footnotetext{
${ }^{1}$ The reason for quotation marks is that the author does not think of philosophy as a 'salon des refusés', a vague discipline 'between theology and science' (Russell) - so much of a definition for an analytic philosopher (!)- but rather as a Kant-Fichte-Schellingian reign of subjectivity, i.e. the will to seek truth; of being and remaining a sujet.
} 
But, there is a logical problem with this defintion: temperature is not 'the average kinetic energy of a system of many particles', it is the average kinetic energy of a system of many particles divided by the Boltzmann constant. The Boltzmann constant is not a dimensionless constant and is meaningless without its dimensions. As the dimensions of the Boltzmann constant are an essential part of the definiens of it and are necessary in the quiddity and what the Boltzmann constant even means, they must not be omitted logically in statements involving this constant; here is where the logical fallacy becomes clear: In the current system of SI units, the units of the Boltzmann constant is Joules/Kelvin, but Kelvin is the base unit of measurement of temperature which means in order to define or understand [temperature] (which is a necessary part of the definiens of temperature), one necessarily has to refer back to the original definiendum temperature. This circular-dependent relation in between the definiens and the definiendum, makes the definition circular. From the perspective of dimensional analysis equation (1) of the definiens reads: [temperature], and our definiendum reads [temperature]. It is true that this is the condition of dimensional analysis: the dimensions of both sides of the equation have to be the same; but there is more to this, for this oughts to be a definition of temperature. There is not a problem at all with the dimensions being equal per se, the problem occurs when one claims this equation as a definition for temperature, because it overlooks the logical necessity of avoidance of circular logic in our definitions. A similar objection holds for entropy. Any intellect familiar with minimums of formal logic can see that this problem is a formal one and cannot be solved by change of units, letting $k_{B}=1$, etc. As such this problem has a deep epistemological root: It is known at least since Euclid[6] (book VII) that to construct any quantitative science one needs a base unit of measurement. As units of measurement are the bedrock of any quantitative discourse, especially physics, any physics that is built upon some base units cannot explain the emergenece of base units themselves; it is evident that to do so would be a logical circle as is the case with the mentioned dogma. Any sane mind can thus see that the oft-repeated justification for 'temperature and entropy are emergent', is based on a circular definition. This serious logical objection undermines the whole 'philosophy' of thermodynamics and statistical mechanics. It is in this light that a rebellion is necessary to chop the root of the problem: entropy is fundamental and single elementary particles posses entropy. But to see this radical proposal we first need to reconcile heat equation with special relativity. As we pointed earlier it is well known that the Heat equation is incompatible with the theory of special relativity[1] for at least one reason: it admits infinite speed of propagation of heat signals within the continuum field. The speed of information propagation is faster than the speed of light in vacuum, which is inadmissible within the framework of special relativity. Consequently heat equation fails to describe situations involving short times, high frequencies and small wavelengths. There is little doubt that heat equation needs to be changed to become compatible with special relativity and observations, but in what manner is the question that we believe is not yet answered satisfactory. Attempts of Hyperbolic heat conduction started by 
Cattaneo[7, 8, 9],

$$
\tau \frac{\partial^{2} T}{\partial t^{2}}+\frac{\partial T}{\partial t}=\chi \nabla^{2} T
$$

where $T: \mathbb{R}^{3} \times \mathbb{R} \rightarrow \mathbb{R}$ is temperature field, try to solve this problem by introducing a relaxation time $\tau$, which is still unsatisfactory for it contains both first and second derivatives, thus treats space and time differently, just as Schrödinger equation does. Maxwell equations in matter are perfect examples of phenomenological equations which are still compatible with the lesson of Minkowski that space and time must be united in a single entity and no law of nature must treat them differently, whether in matter or vacuum, whether fundamental or not. Nobody considers the $\mathbf{D}$ and $\mathbf{H}$ fields fundamental in any sense; that is why their corresponding equation are called macroscopic equations, yet the equations governing $\mathbf{D}$ and $\mathbf{H}$ still satisfy Minkowski's maxim. All the existing attemps of reconciling heat equation with special relativity focus on heat conduction, while there is an important problem which will not be solved by any manipulation of the heat equation: I maintain that there is no satisfactory theory of thermal radiation in vacuum. In vacuum there is no material substance which can transfer heat and all the descriptions using the energy of electromagnetic field are superficial because they confuse heat and light. If thermal radiation is the same as the energy of electromagnetic field, then what is the necessity of using heat equation for conduction? why not continue with the assumption that heat is the same as the energy of electromagnetic field even in the realm of material media and therefore it should be described by the macroscopic Maxwell equations? Such division between the modes of explanation of heat cannot be right for the simple reason that heat is one and the same phenomenon in the entire universe, be it in vacuum or matter. Accordingly a unified picture of heat transfer is required which will reduce to

$$
\nabla^{2} T-\frac{1}{c^{2}} \frac{\partial^{2} T}{\partial t^{2}}=0
$$

in vacuum in absence of sources. To find such equation we use the wellknown analogy between Fourier's law

$$
\mathbf{q}=k \mathbf{C}=-\kappa \nabla T,
$$

and Ohm's law

$$
\mathbf{J}=\sigma \mathbf{E}=-\sigma \nabla \varphi,
$$

as our guiding principle. This analogy is well supported empirically ${ }^{2}$ : just as there corresponds to Ohm's law the Hall effect, one might expect that a thermal analogue also exists which in fact does. By this analogy we can immediately see that temperature is the potential function of the field of heat; i.e. if by $\mathbf{C}$ we denote the vector field of heat ${ }^{3}$, we have

$$
\mathbf{C}=-\nabla T \text {. }
$$

\footnotetext{
${ }^{2}$ According to the philosophical views of the author, apart from the empiricist perspective on analogies, there is also an epistemological aspect to analogies; as a degenerate outline of my ideas in this regard see[10].

${ }^{3}$ Standing for Calor, latin for heat.
} 
From this perspective we realise that any attempt of manipulating the heat equation is misguided: we do encounter this equation

$$
\frac{\partial \mathbf{B}}{\partial t}=\frac{1}{\mu_{0} \sigma} \nabla^{2} \mathbf{B}
$$

in the realm of magnetohydrodynamics ${ }^{4}$ yet no one ever tries to modify this equation to make it compatible with special relativity because first, it is only an approximation and the exact equations are indeed compatible with special relativty; second, retrospectively it is just not the right approach. The right approach is to use $\mathbf{D}$ and $\mathbf{H}$ fields. Consider this historical thought experiment: Suppose history of physics unfolded in an alternative way such that this equation was discovered before Maxwell equations. By the rationale which drives manipulations of heat equation, we should have manipulated this equation too, which in retrospective is utterly misguided and could only lead us astray.

Apart from what the mentioned analogy suggests, namely that just as we have a field theory for electromagnetism we must have one as well for heat, it is well-established that one major aspects of the paradigm of special relativity is the importance of field theories. We expect a field equation for temperature field $T$ which recognises $\hbar$, for it was efforts of understanding heat (radiation) that led Planck to the quanta hypothesis hence later quantum theory. Quantum theory clearly manifests itself in thermal radiation, so any modification of heat equation which does not takes $\hbar$ into account cannot be considered a deep progress.

By benefit of hindsight, we know the best approach to electromagnetism is to first do it in vacuum which is simple because we are only facing essential fundamental constituents and need not worry about emergent complexities of materials like polarisation. It would then be straightforward to do electromagnetism in media by introducing electric and magnetic polarisation vectors. In this paper, we shall follow a heuristic approach which helps to recognise the continuity of scientific pursuit and is fair to historical evolution of ideas, but at the end we can step back and infer possible ontological consequences.

In order to find a wave equation for $T$ field, we must first try to see how a field theory is constructed: field theories usually rely on a wave equation in Lorenz gauge; the only characteristic of a wave is its speed of propagation and by Einstein's principle of constancy of velocity of light, all fields in vacuum propagate at the speed of light. The mathematical implementation of field theories is best done via the formalism of four-potentials, and this formalism is even applicable to Newtonian gravity resulting in $\square \phi=-4 \pi G \rho$. In this formalism one adds the relevant material source of fields (e.g. mass density for gravity, charge density for electrostatics, etc.) to the source term $J^{\mu}$ and by applying Maxwell equation $\square A^{\mu}=\mu_{0} J^{\mu}$

${ }^{4}$ This is the induction equation of magnetohydrodynamics

$$
\frac{\partial \mathbf{B}}{\partial t}=\frac{1}{\mu_{0} \sigma} \nabla^{2} \mathbf{B}+\nabla \times(\mathbf{v} \times \mathbf{B})
$$

in the diffusive limit, i.e. for very small magnetic Reynolds numbers. 
arrives at the desired field equation. For example in case of gravity,

$$
J^{0}=c \sqrt{4 \pi \epsilon_{0} G} \rho
$$

and

$$
A^{0}=-\frac{\phi\left(x^{\rho}\right)}{c \sqrt{4 \pi \epsilon_{0} G}},
$$

where $\phi$ is the Newtonian gravitational potential. Needless to say that one must always pay attention to dimensional analysis so that the relevant potential is multiplied by proper constants to match the dimension of electric current density and magnetic vector potential. For gravity, one can find those constants by dimensional analysis, except for $4 \pi$ factor and this indicates that the correct constant of gravitoelectromagnetism is $\eta_{0}=\sqrt{4 \pi \epsilon_{0} G}$, therefore we will use $\eta_{0}$ in all of our dimensional-analytic considerations.

\section{Wave Equation for Temperature Scalar Field}

In light of the discussion of previous section, our task is simple: We should add the temperature scalar field to the four-potential of electromagnetism. A dimensional analysis using all the known fundamental constants of physics $^{5}$ shows that the right constant whose multiplication with $T$ will make for it possible to sit in the four-potential of electromagnetism is

$$
\zeta_{0}:=\frac{k_{B}}{\sqrt{4 \pi \epsilon_{0} \hbar c^{3}}}
$$

thus,

$$
A^{0}:=\zeta_{0} T=\frac{k_{B}}{\sqrt{4 \pi \epsilon_{0} \hbar c^{3}}} T\left(x^{\rho}\right) .
$$

The vital question that we must now answer is that what is the source term for a wave equation for T? Before answering this question we must justify our use of a local temperature field: we are seeking an equation which describes variations in temperature field, something that is not possible in equilibrium thermodynamics in which we only consider systems in thermal equilibrium characterised by uniform temperature fields, no heat flow and zero entropy production. Evidently we are entering the realm of non-equilibrium thermodynamics which is a well-developed discipline, but we need not go to details of that field here if we restrict our discourse to Classical Irreversible Thermodynamics according to its fundamental hypothesis of local equilibrium[11], all the thermodynamical variables defined in equilibrium, are defined outside of equilibrium as well but vary with spacetime. The only other result from Classical Irreversible Thermodynamics we shall need is an indirect result from Onsager's work[12] according to which, by the assumption of microscopic reversibility, we have a conservation law for entropy

$$
\frac{\partial \sigma}{\partial t}+\nabla \cdot \mathbf{J}=0
$$

${ }^{5} G, \mu_{0}, \epsilon_{0}, k_{B}$, and $\hbar$. 
where $\sigma$ is entropy density per volume, and we call $\mathbf{J}$ entropy current with dimensions of $[$ mass $][\text { time }]^{-3}[\text { temperature }]^{-1}$.

As the source term for a scalar field must itself be a scalar we conclude that $\sigma$ is the source for $T$ field. $\mathbf{J}$ is clearly signaling existence of a new field as for a moving source of entropy (with entropy density $\sigma$ ) one has

$$
\mathbf{J}=\sigma \mathbf{v}
$$

To find a wave equation for $T$ it only remains to divide $\sigma$ by the proper constant $\zeta_{0}$, to make its addition to the source term of electromagnetism possible:

$$
J^{0}:=\sigma / \zeta_{0}=\frac{\sqrt{4 \pi \epsilon_{0} \hbar c^{3}}}{k_{B}} \sigma\left(x^{\rho}\right) .
$$

We now apply Maxwell equation (in Lorenz gauge) $\square A^{\mu}=\mu_{0} J^{\mu}$, to arrive at

$$
\square T=\frac{4 \pi \hbar c}{k_{B}^{2}} \sigma
$$

This step was necessary to find the right constant $4 \pi \hbar c / k_{B}^{2}$, but now that we have the wave equation for $T$ in Lorenz gauge we can proceed to find 'un-gauged' field equations.

\section{Covariant theory}

\section{$3.1 \quad$ Onsager four-vector}

Four-potential formalism requires a four-current as source term. Source terms of field theories all satisfy a conservation law of the form of

$$
\partial_{\mu} J^{\mu}=0
$$

Introducing Onsager four-vector

$$
\sigma^{\mu}:=(\sigma, \mathbf{J} / c),
$$

where $c$ is the speed of light in vacuum, Onsager's Conservation of entropy now reads

$$
\partial_{\mu} \sigma^{\mu}=0
$$

just as required for contruction of a field theory.

\subsection{Constructing Temperature four-vector}

As Onsager four-vector is the source term for temperature field, we infer that a new vector field exists in analogy to the magnetic vector potential, which we denote by $\mathbf{R}$. Therefore, we define four-temperature by

$$
T^{\mu}:=(T, \mathbf{R} / c)
$$

where $c$ is the speed of light in vacuum. Judging by dimensions,

$$
\left[R^{a}\right]=[\text { Temperature }][\text { Speed }],
$$

this new vector potential is not entirely unknown and is called Kinematic heat flux; its dynamics will have predictions about thermodynamics of moving bodies which we will consider later. 


\subsection{Onsager field tensor}

To find the lagrangian density of heat field, we now define Onsager tensor by

$$
\mathcal{O}_{\mu \nu}:=\partial_{\mu} T_{\nu}-\partial_{\nu} T_{\mu}
$$

and its dual by

$$
\tilde{\mathcal{O}}^{\mu \nu}=\frac{1}{2} \epsilon^{\mu \nu \rho \sigma} \mathcal{O}_{\rho \sigma}
$$

\subsection{Lagrangian density}

Using onsager tensor defined above, the lagrangian density is

$$
\mathcal{L}=-\frac{k_{B}^{2}}{8 h c} \mathcal{O}_{\mu \nu} \mathcal{O}^{\mu \nu}-T^{\rho} \sigma_{\rho}=\mathcal{L}\left(T_{\alpha}, \partial_{\beta} T_{\alpha}\right)
$$

Application of Euler-Lagrange equations yields the field equations

$$
\partial_{\mu} \mathcal{O}^{\mu \nu}=\frac{2 h c}{k_{B}^{2}} \sigma^{\nu} \quad \text { and } \quad \partial_{\mu} \tilde{\mathcal{O}}^{\mu \nu}=0
$$

\section{Implications}

It is now time to step back and look at the theory we have and see what it is telling us. As our starting point was entirely based on special-relativistic considerations, the two famous problems that we mentioned in the introduction are completely resolved by (4), viz.

\subsection{Temperature and Entropy of a moving body}

As both temperature and entropy are added to four-vectors in our theory, in Minkowski spacetime they both transform according to the usual rules of transformations of four-vectors, viz.

$$
T^{\prime \mu}=\Lambda_{\nu}^{\mu} T^{\nu}, \quad \sigma^{\prime \mu}=\Lambda_{\nu}^{\mu} \sigma^{\nu}
$$

where $\boldsymbol{\Lambda}$ is the Lorentz transformation matrix.

\subsection{Entropy of single particles}

In both of the two established classical field theories (gravity and electromagnetism), the source terms correspond to fundamental characteristic properties of single particles (mass and electric charge respectively ${ }^{6}$ ). By inductive reasoning we propose

Principle 1. Single particles possess entropy.

\footnotetext{
${ }^{6}$ If we want to have the duality invariance of the Maxwell equations by adding magnetic charges, this observation would be even more well-founded.
} 
Principle 2. Rest-cold-energy ${ }^{7}$ of a particle is given by

$$
E=T_{P} S_{0}=\sqrt{\frac{\hbar c^{5}}{G k_{B}^{2}}} S_{0}
$$

For example, entropy of a single electron in an interaction that inertial energy $\left(m c^{2}\right)$ is completely transformed to thermal energy is

$$
S_{e}=m_{e} c^{2} \sqrt{\frac{G k_{B}^{2}}{\hbar c^{5}}} \approx 5.7 \times 10^{-46} \text { Joules/Kelvin } .
$$

As another justification for above principles consider the following

\subsubsection{Induction on de Broglie's methodology}

We believe that a proposal which is not forbidden logically or empirically, must be proposed and seriously considered. Historically, this was a huge success with de Broglie's proposal. Retrospectively what de Broglie did was to use conservation of energy

$$
m c^{2}=h \nu
$$

to say that anything that can have mass must have frequency. There must exist a deeper reason why this ontological extrapolation of de Broglie worked. We call such 'deeper reason' the generalizability of energy, according to which, any property which can be attributed through a conservation of energy is ontologically justified. According to this supposed property of energy, similar to de Broglie's proposal, through

$$
m c^{2}=S T_{P}
$$

anything that can have mass must have entropy, and since there is no logical or empirical obstacle to assuming that entropy can be associated with a single particle, it must be assumed to be so.

\subsection{Principle of Maximum Temperature}

If (9) is true, $m_{0} c^{2}=S_{0} T_{P}$ suggests that just as $c$ is a maximum of nature, $T_{P}$ must be a maximum too. Accordingly we propose

Principle of Maximum Temperature Maximum temperature attainable in the universe (in vacuum and inertial frames) is the Planck temperature $T_{P}$, which in terms of fundamental constants is given by

$$
T_{P}=\sqrt{\frac{\hbar c^{5}}{G k_{B}^{2}}} .
$$

\footnotetext{
${ }^{7}$ Rest-energy is familiar to us, but as we shall see, there is another kind of energy which we call cold-energy which does exist even for systems with no relative motion. Therefore rest-cold-energy means the energy of a particle at rest at $T=0$.
} 
Mathematical implementation of this principle $\left(T \leq T_{P}\right)$ is done by introducing theta factor

$$
\theta:=\frac{1}{\sqrt{1-\frac{T}{T_{P}}}}
$$

Now consider two systems $A$ and $B$ with no relative motion, with non-zero temperatures $T_{A}$ and $T_{B}$ and $T_{A} \neq T_{B}$. We ask what is the entropy of system $B$ as perceived by system $A$ ? This question might sound strange because we are used to absolute statistical mechanics, in which we compare different systems without realising that entropy is a local and relative quantity, but in light of (10) this question is just the right question that must be asked ${ }^{8}$.

To answer the question, notice that (10) suggests that one cannot associate a universal temperature to an object, i.e. there is no absolute notion of temperature, just like there is no absolute notion of velocity, so whenever we are asked 'what is the temperature of that object?' we must ask with respect to which system? We have long been aware of this relativity of temperature by saying that only temperature differences matter.

Thus we first define the relative temperature of $A$ and $B$ by

$$
T:=\left|T_{A}-T_{B}\right|
$$

Then (10) implies that the entropy of $B$ perceived by $A$ is given by

$$
S^{\prime}=\frac{1}{\sqrt{1-\frac{T}{T_{P}}}} S,
$$

meaning that temperature difference causes two systems to disagree on entropy of one another, viz. $A$ measures the entropy of $B$ to be reduced by a factor of $\theta$ than what $B$ itself measures.

In particular these considerations mean that the notion of entropy of universe is meaningless unless the universe is in uniform thermal equilibrium, viz.

$$
d T^{\mu}=0
$$

everywhere on spacetime.

To the best of my knowledge the proposed theta factor (10) is new. There have already been attempts[13, 14] that use the factor

$$
\tilde{\theta}=\frac{1}{\sqrt{1-\frac{T^{2}}{T_{P}^{2}}}} .
$$

In light of (9) it is easy to see that the above factor is wrong, as we expect any proper theory to yield fundamental results of the statistical

\footnotetext{
${ }^{8}$ There is a possibility of confusion here because we are only accustomed to transformations which involve velocity (Lorentz transformations), thus subconsciously the picture of two observers which move relative to one another is conjured up. One can use a 'trick' to think about this problem: Think of temperature as velocity; two systems with different temperatures are like two particles which move relative to one another. Just like the two particles disagree about eachother's mass, two systems with different temperatures disagree about eachother's entropy.
} 
mechanics, a major one being the equipartition theorem:

$$
E=\frac{1}{2} k_{B} T
$$

consider a hot atom with entropy $k_{B}$. According to our proposal the hot energy of this atom must be

$$
E=(\theta-1) T_{P} S=(\theta-1) T_{P} k_{B} \approx \frac{1}{2} k_{B} T
$$

where we have used the Taylor approximation for the theta factor. Our proposal is therefore naturally compatible with the equipartition theorem. Now consider the other factor $\tilde{\theta}$ for the same atom:

$$
E=(\tilde{\theta}-1) T_{P} S=(\tilde{\theta}-1) T_{P} k_{B} \approx \frac{1}{2} k_{B} \frac{T^{2}}{T_{P}},
$$

in sheer contradiction with the equipartition theorem.

\subsection{Corrections to the equipartition theorem}

We showed that $E=(\theta-1) T_{P} S$ yields one of the key results of statistical mechanics (equipartition theorem) as the first term in its Taylor expansion. We can continue with the Taylor expansion

$$
E=\frac{1}{2} k_{B} T+\frac{3}{8} \frac{k_{B}^{2}}{E_{P}} T^{2}+\mathcal{O}\left(T^{3}\right),
$$

to see that the equipartition theorem has to be corrected, especially in the realm of quantum gravity.

\section{Conclusion}

In this paper we proposed a complete theory of heat that decisively solves all the mentioned problems; but the cost of this elegant resolution is the abandonment of yet another sacred cows of academia: emergence of temperature and entropy, and this is exactly what history has taught us: elegant over-arching resolutions come with radical conceptual consequences.

\section{References}

[1] E. R. G. Eckert and Drake R. M. Analysis of Heat and Mass Transfer. Tokyo: McGraw-Hill, 1972.

[2] Christian Farías et al. What is the temperature of a moving body? Scientific Reports, 7(17657), 2017.

[3] F. Reif. Fundamentals of Statistical and Thermal Physics. McGrawHill, 1965.

[4] David Chandler. Introduction to Modern Statistical Mechanics. Oxford University Press, 1987. 
[5] Charles Kittel and Herbert Kroemer. Thermal physics. New York: W.H. Freeman, 1980.

[6] Euclid. Euclid's Elements. Green Lion Press, 2002.

[7] C. R. Cattaneo. Sur une forme de l'équation de la chaleur éliminant le paradoxe d'une propagation instantanée. Comptes Rendus, 247(4):431, 1958.

[8] P. Vernotte. Les paradoxes de la theorie continue de l'équation de la chaleur. Comptes Rendus, 246(22):3154, 1958.

[9] M. Chester. Second sound in solids. Physical Review, 131(15):20132015, 1963

[10] Radin Dardashti et al. Confirmation via analogue simulation: What dumb holes could tell us about gravity. The British Journal for the Philosophy of Science, 68(1), 2017.

[11] Georgy Lebon and David Jou. Understanding Non-equilibrium Thermodynamics. Springer-Verlag Berlin Heidelberg, 2008.

[12] L. Onsager. Reciprocal relations in irreversible processes. i. Physical Review, 131(15):2013-2015, 1931.

[13] Zhao Liu. Thermal relativity. Communications in Theoretical Physics, 56(6), 2011.

[14] Carlos Castro Perelman. Thermal relativity, modified hawking radiation, and the generalized uncertainty principle. International Journal of Geometric Methods in Modern Physics, 16(10), 2019. 